\title{
sciendo
}

\section{Comparative Study of Kinematics and Muscle Activity Between Elite and Amateur Table Tennis Players During Topspin Loop Against Backspin Movements}

\author{
by \\ Meizi Wang ${ }^{1}$, Lin Fu ${ }^{1}$, Yaodong $\mathrm{Gu}^{1}$, Qichang Mei ${ }^{1,2,3}$, Fengqin Fu', \\ Justin Fernandez $z^{2,3}$
}

\begin{abstract}
This study investigated differences of lower limb kinematics and muscle activity during table tennis topspin loop against backspin movements between elite players (EPS) and amateur players (APs). Ten EPs and ten APs performed crosscourt backhand loop movements against the backspin ball with maximal power. Vicon motion analysis and a MEGA ME6000 system was used to capture kinematics and surface EMG data. The motion was divided into two phases, including the backswing and swing. The joints' flexion and extension angle tendency between EPs and APs differed significantly. The coefficient of multiple correlation $(C M C)$ values for EPs were all beyond 0.9, indicating high similarity of joint angles change. APs presented moderate similarity with CMC values from 0.5 to 0.75 . Compared to $A P_{S}$, EPs presented larger ankle eversion, knee and hip flexion at the beginning moment of the backswing. In the sEMG test, EPs presented smaller standardized AEMG (average electromyography) of the lower limb muscles in the rectus femoris and tibia anterior on both sides. Additionally, the maximum activation of each muscle for EPs was smaller and MPF (mean power frequency) of the lower limb was greater during the whole movement. The present study revealed that EPS could complete this technical motion more economically than APs, meanwhile, EPs were more efficient in muscle usage and showed better balance ability.
\end{abstract}

Key words: kinematics, EMG, backhand loop, skilled level, table tennis.

\section{Introduction}

Analysis of the skills and tactics of professional Chinese table tennis players reveals that the backhand loop is not only a primary technique, but also a key movement for scoring. Backhand racket motions against topspin and backspin incoming balls are different. For example, the incoming topspin ball tends to be returned hit upward to counter the spin itself, while the incoming backspin ball tends to be returned by hitting downward for the same reason. Incoming backspin balls tend to be returned higher and the player must adjust the angle of the racket surface appropriately according to the amount of pre-impact ball spin (Neal, 1989). The opponent has less opportunity to hit an offensive stroke against a ball with higher speed and greater spin. Therefore, it is important to control the backhand backspin strokes and hit the stroke with a high racket speed.

Tsai et al. (2010) reported that high-level table tennis players would increase the racket tilt and swing with an upward angle when spinning down the forehand. However, less attention has been paid to the biomechanics of a table tennis

1 - Faculty of Sports Science, Ningbo University, Ningbo, China.

2 - Department of Engineering Science, University of Auckland, Auckland, New Zealand.

3 - Auckland Bioengineering Institute, University of Auckland, Auckland, New Zealand. 
backhand investigating the contribution of lower limb rotation to the racket speed in the backhand shots of advanced players (Huang, 2013; Iino and Kojima, 2015). This important feature has been demonstrated in a case study of a professional tennis player highlighting the effect of the lower limb on improving stroke performance (Endo, 2006).

The sEMG analyses of the tennis serve have been used to detect patterns of muscle activation in the lower trunk (Chow et al., 2003). Tsai et al. (2010) found that the value of integrated electromyography of a forehand backspin was larger than a forehand forespin. Wan (2015) found that the tibialis anterior muscle was activated first during the push up with the leg muscles, and followed by the waist, shoulder, wrist, hand, finally completing the entire hitting action. The performance level has been proven to be linked with movement characteristics in our previous studies (Fu et al., 2016; Qian et al., 2016). This study highlights the importance of coordination between the various muscles and how knowledge of kinematics and muscle activity helps understand how to accelerate the distal segment effectively.

The purpose of the current study was to investigate the kinematics and muscle activity of the table tennis topspin loop against backspin movements between elite (EPs) and amateur players (Aps). This may provide a theoretical basis for training of athletes. It has been previously reported that advanced players mainly exploited lower trunk axial rotation to generate higher racket speed at impact (Iino and Kojima, 2015). Therefore, we hypothesized that the average value of the electromyogram and the rate of angle change of EPs would be larger compared to APs during the whole technical movement.

\section{Methods}

\section{Participants}

Twenty male table tennis players (ten EPs and ten APs) participated in this study. Participants were asked to perform a crosscourt backhand loop against the backspin ball with maximal power. All participants were righthanded, had no previous lower extremity and foot diseases or deformity, and were free from injury for at least six months prior to the test. The years of practice between EPs and APs showed a significant difference. All subjects were asked to wear training shoes and were informed of the test procedures and the dropping position of the ball. The study was approved by the Ethics Committee of the University.

\section{Design and Procedures}

An eight-camera Vicon motion analysis system (Oxford Metrics Ltd., Oxford, UK) was used to capture lower limb kinematics with a frequency of $200 \mathrm{~Hz}$. Participants were required to wear tight-fitting pants and 16 reflective markers (diameter: $14 \mathrm{~mm}$ ) were attached to the left and right lower limb, respectively. The marker locations included: anterior-elite iliac spine, posterior-elite iliac spine, lateral mid-thigh, lateral knee, lateral mid-shank, lateral malleolus, second metatarsal head and calcaneus. Each trial started with EMG data collection, followed by kinematic data collection. EMG and kinematic data from separate trials were needed because EMG electrodes interfered with reflective marker placement on the lower back.

After five minute jogging on a treadmill as a warm-up, surface electrodes were attached to selected muscles of the lower trunk, including the left rectus femoris (LRF), right rectus femoris (RRF), left anterior tibialis (LTA), and right tibia anterior (RTA). The skin surface where the electrodes was located was cleaned with alcohol and shaved when necessary. Electrodes were placed over the belly of each muscle parallel to the muscle's line of action with a centre-to-centre distance of $2.5 \mathrm{~cm}$. The MEGA ME6000 system was used to record electromyographic signals. AEMG, MPF and MF would increase following an increase in the level of muscle contraction (Romanliu, 2016). To obtain maximum EMG levels, two maximal isometric contractions were performed before the experimental trials. In all trials, the participants served with effort comparable to their serves during competition. In the batting test, athletes performed in accordance with a self-selected action to moderate the ball, which was sent by a special table tennis ball machine previously calibrated; each ball was sent every $20 \mathrm{~s}$ and the test was repeated five times.

Tests were conducted in a table tennis training gymnasium of the University. The floor was made of wood which was consistent with training and competition courts. The ball machine was placed $1.2 \mathrm{~m}$ away from the opponent's court 
and it was used to project backspin balls directly to the backside of the subjects' court. Settings of the machine including a projecting angle, a radian angle, velocity, and frequency were consistent for all balls. Sufficient time was given for participants to warm up and familiarize themselves with the measuring instrument. Since players were proficient in backhand backspin loop technique, only a brief instruction was needed to ensure the motion quality. During testing, participants were asked to perform a single crosscourt backhand backspin loop with maximal effort. At least five successful trials were performed for each subject. Smoothness of foot motion was judged by players themselves and the quality of the balls' effect was supervised by their coaches. Data were collected separately for the five trials to distinguish between consecutive strokes.

\section{Data analysis}

Kinematic data, including angle variation range and instantaneous angles for the ankle, knee and hip in the sagittal, coronal and horizontal planes were recorded. The sEMG data including AEMG, MPF and MF were included. All statistical analyses were conducted using SPSS version 19.0 software (SPSS Inc., Chicago, IL, USA) for Windows. Initial Shapiro-Wilk tests validated that the data were normally distributed. To examine the differences between two skilled levels, independent sampled t-tests were taken for each dependent variable. The significance level for all tests was set at 0.05 .

The similarity of the curves was evaluated by the coefficient of multiple correlation (CMC), which was applied to the change of the joint angle when performing the topspin loop against the backspin. The CMC was calculated as:

$$
\sqrt{1-\frac{\sum_{i=1}^{m} \sum_{i=1}^{n}\left(Y_{i f}-\bar{Y}_{f}\right)^{2} / n(m-1)}{\sum_{i=1}^{m} \sum_{i=1}^{n}\left(Y_{i j}-\bar{Y}\right)^{2} f(n m-1)}}
$$

In the formula: $m$ represents the number of curves with $m_{E P}=10$ and $m_{A P}=10 ; n$ represents the number of data of each curve; $Y_{i j}$ is the $j$ th data of the $i$ th curve; $\bar{Y}_{j}$ is the average of the $j$ th data of all curves, $\bar{Y}$ is the mean of $n$ data for all curves. The closer the CMC is to 1 , the higher the similarity of the curve. The following definitions were used in this study: no similarity for between 0 and 0.25 , low similarity for 0.25 to 0.5 , moderate similarity for 0.5 to 0.75 , and high similarity for more than 0.75 values following Maszczyk et al. (2014), Li et al. (2011), Yu (2003) and Kadaba et al. (1989).

The magnitude of the EMG signals was transformed into a muscle activation variable, ai (where $i$ represented each muscle in the model) and it was a time varying value with a magnitude between 0 and 1 (max). The raw EMG signal had low frequency noise removed with a cut-off frequency of $10 \mathrm{~Hz}$ and then rectified. This filter was implemented in Matlab. The rectified EMG signal was normalized by dividing the peak rectified EMG value obtained during a maximum voluntary contraction (MVC), and then applying a low-pass filter to the resultant signal (Buchananet al., 2004; Gołaś et al., 2017).

\section{Results}

The flexion and extension of the EPs and APs' joint angles showed a significant difference, and the CMC values of Eps were all beyond 0.9 (Figure 1). Changes in their joint angles showed high similarity when performing the topspin loop against the backspin movement, and the curves of APs were located in the range of moderate similarity (CMC from 0.5 to 0.75 ).

Figure 2 illustrates the lower limb joint angle in the coronal plane at the beginning of the backswing moment. EPs showed more dorsiflexion, hip adduction and less knee adduction than APs.

Table 1 shows joint angles for the ankle, knee and hip between EPs and APs at the beginning of the backswing movement. Joint angles at the beginning moment were significantly different in the sagittal, coronal and horizontal planes (except the knee joint in the horizontal plane) between EPs and APs. Compared to APs, the ankle angle of EPs showed significantly larger dorsiflexion, eversion and external rotation (dorsiflexion: $p<$ 0.05 , eversion: $p<0.05$, external rotation: $p<0.05$ ), the knee joint of EPs showed larger flexion and abduction (flexion: $p<0.05$, external rotation: $p<$ $0.05)$, the hip joint of EPs showed greater flexion, adduction and external rotation (flexion: $p<0.05$, adduction: $p<0.05$, external rotation: $p<0.05$ ).

However, Table 2 exhibits lower limb joints, including the ankle, knee and hip at the end 
moment of the swing during topspin loop against backspin movement. There were also significant differences of three joints at this moment. In contrast to EPs, APs showed less dorsiflexion, knee flexion and greater hip flexion in the sagittal plane (dorsiflexion: $p<0.05$, flexion $p<0.05$ ). EPs showed larger hip abduction than APs (abduction $p<0.05)$ in the coronal plane, additionally, EPs showed ankle inversion and knee adduction while APs showed ankle eversion and knee abduction. In the horizontal plane, EPs presented internal rotation, while APs showed external rotation in three joints. The differences in this plane were significant.

Table 3 shows the rate of angle change between EPs and APs during the swing movement. The rate of change of Eps' joint angles during backswing movement was significantly different from APs in the sagittal, coronal and horizontal planes of joints (beside the ankle joint in the coronal plane) $(p<0.05)$.

Lower limb muscle standardization average electromyography (iAEMG) between EPs and APs was computed for the backhand backspin loop movement (Figure 3). The standardized iAEMG of the lower limbs of EPs on both sides of the rectus and tibialis anterior muscles was smaller compared to APs, particularly on the left side of the rectus femoris and the right side of the tibialis anterior muscle.

MPF of the lower limb showed a significant difference between EPs and APs in the whole technical movement of the backhand backspin loop, and MPF of the lower limbs of EPs was greater than that of APs (LRF: $p<0.05$, RRF: $p<$ 0.05 , LTA: $p<0.05$, RTA: $p<0.05$; Picture 1).

\begin{tabular}{|c|c|c|}
\hline \multirow{2}{*}{\multicolumn{3}{|c|}{$\begin{array}{r}\text { Table } \mathbf{1} \\
\text { The angle at beginning of the backswing between EPS and APs (degree) }\end{array}$}} \\
\hline & & \\
\hline & $\mathrm{EP}$ & $\mathrm{AP}$ \\
\hline \multicolumn{3}{|l|}{ Variables of the right leg } \\
\hline Ankle dorsiflexion/plantarflexion (-) & $22.34 \pm 3.38^{*}$ & $9.41 \pm 1.78$ \\
\hline Ankle inversion/eversion (-) & $2.73 \pm 0.57^{*}$ & $-3.82 \pm 0.86$ \\
\hline Ankle internal rotation/external rotation (-) & $-7.59 \pm 1.86^{*}$ & $18.05 \pm 4.90$ \\
\hline Knee flexion/knee extension (-) & $40.24 \pm 6.24^{*}$ & $19.90 \pm 3.18$ \\
\hline Knee adduction/knee abduction (-) & $28.41 \pm 3.95^{*}$ & $-8.36 \pm 2.31$ \\
\hline Knee internal rotation/knee external rotation (-) & $-11.38 \pm 3.78$ & $-10.85 \pm 3.84$ \\
\hline Hip flexion/hip extension (-) & $34.75 \pm 2.34^{*}$ & $25.82 \pm 4.46$ \\
\hline Hip adduction/hip abduction (-) & $-25.14 \pm 5.84^{*}$ & $-11.67 \pm 1.47$ \\
\hline Hip internal rotation/hip external rotation (-) & $8.10 \pm 2.04^{*}$ & $-23.92 \pm 5.98$ \\
\hline \multicolumn{3}{|c|}{$\begin{array}{c}\text { * significant differences in the ankle, hip and knee joint. } x, y, z \text { represent sagittal, } \\
\text { coronal, and horizontal planes, respectively. }\end{array}$} \\
\hline
\end{tabular}

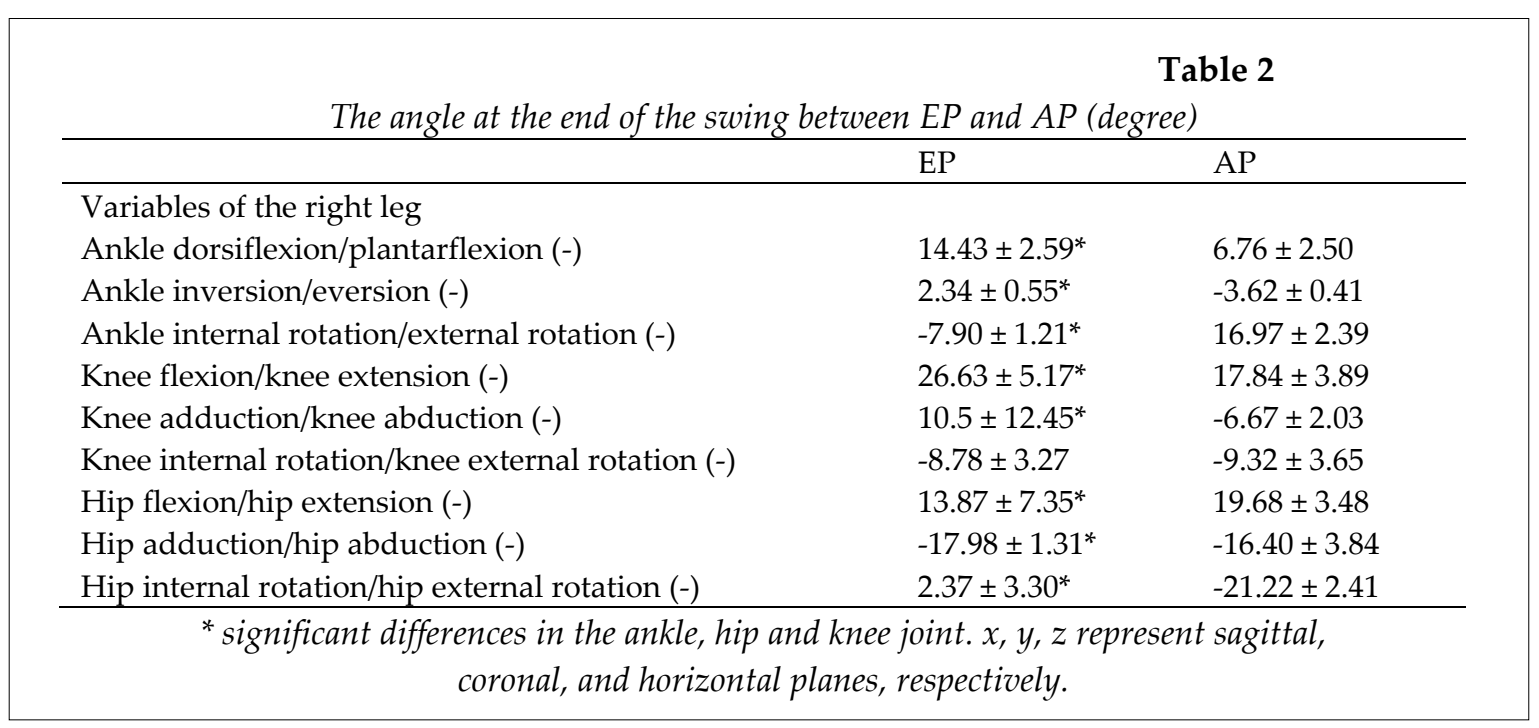


Table 3

The rate of angle change between EP and AP during the swing movement (degree/s).

\begin{tabular}{|c|c|c|c|c|c|c|}
\hline & \multicolumn{2}{|c|}{ Ankle } & \multicolumn{2}{|c|}{ Knee } & \multicolumn{2}{|c|}{ Hip } \\
\hline & $\mathrm{EP}$ & $\mathrm{AP}$ & EP & $\mathrm{AP}$ & EP & $\mathrm{AP}$ \\
\hline Sagittal & $1.01 \pm 0.13^{*}$ & $\begin{array}{c}0.22 \pm \\
0.09\end{array}$ & $2.84 \pm 0.37^{*}$ & $0.41 \pm 0.15$ & $0.94 \pm 0.14^{*}$ & $0.29 \pm 0.08$ \\
\hline Coronal & $0.05 \pm 0.02$ & $\begin{array}{c}0.06 \pm \\
0.01\end{array}$ & $0.86 \pm 0.09^{*}$ & $0.07 \pm 0.01$ & $1.13 \pm 0.24^{*}$ & $0.24 \pm 0.05$ \\
\hline Horizontal & $0.18 \pm 0.06^{*}$ & $\begin{array}{c}0.38 \pm \\
0.06\end{array}$ & $1.00 \pm 0.1^{*}$ & $0.11 \pm 0.03$ & $0.39 \pm 0.11^{*}$ & $0.11 \pm 0.03$ \\
\hline
\end{tabular}

* differences in the ankle, hip and knee joint.

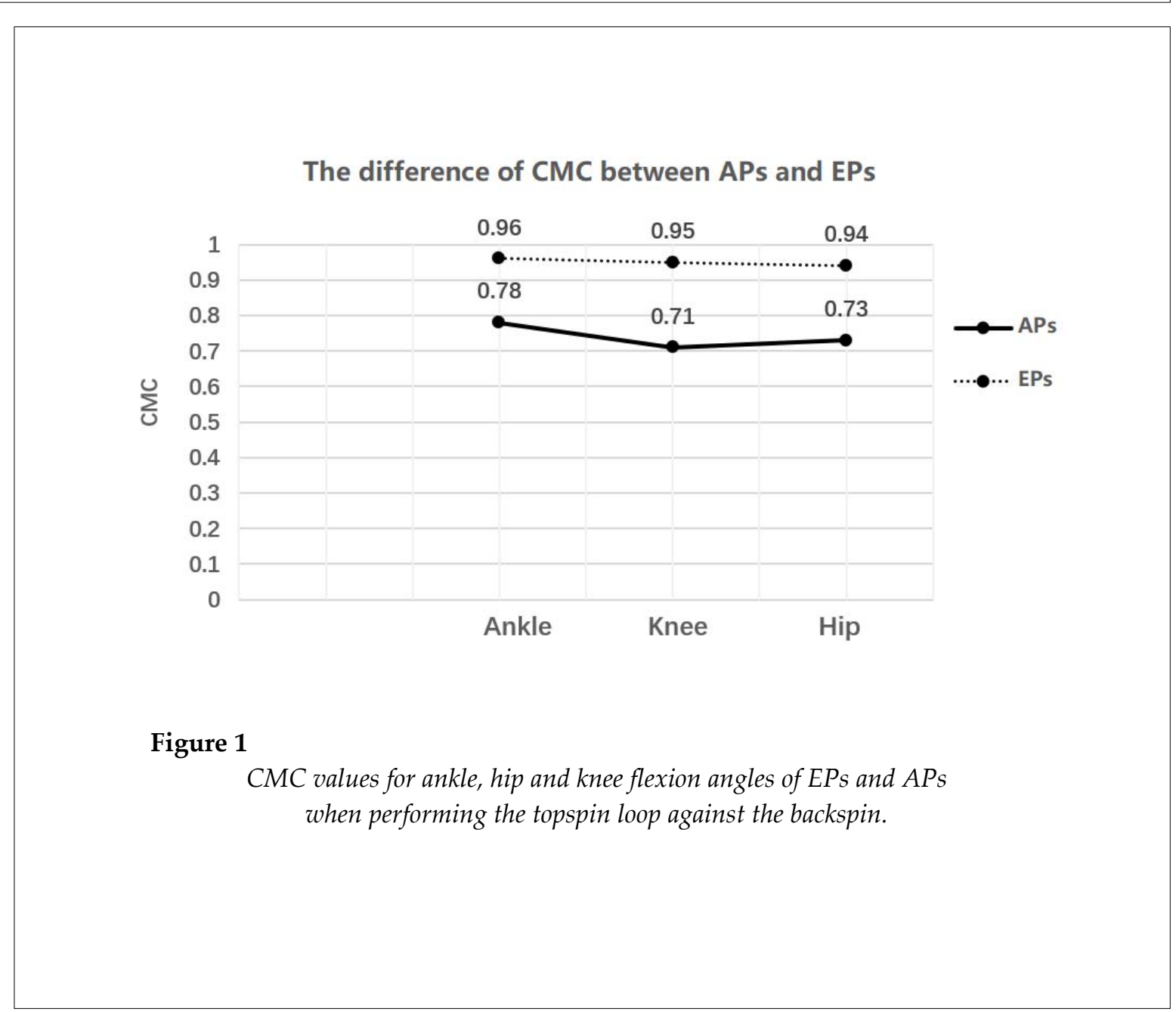



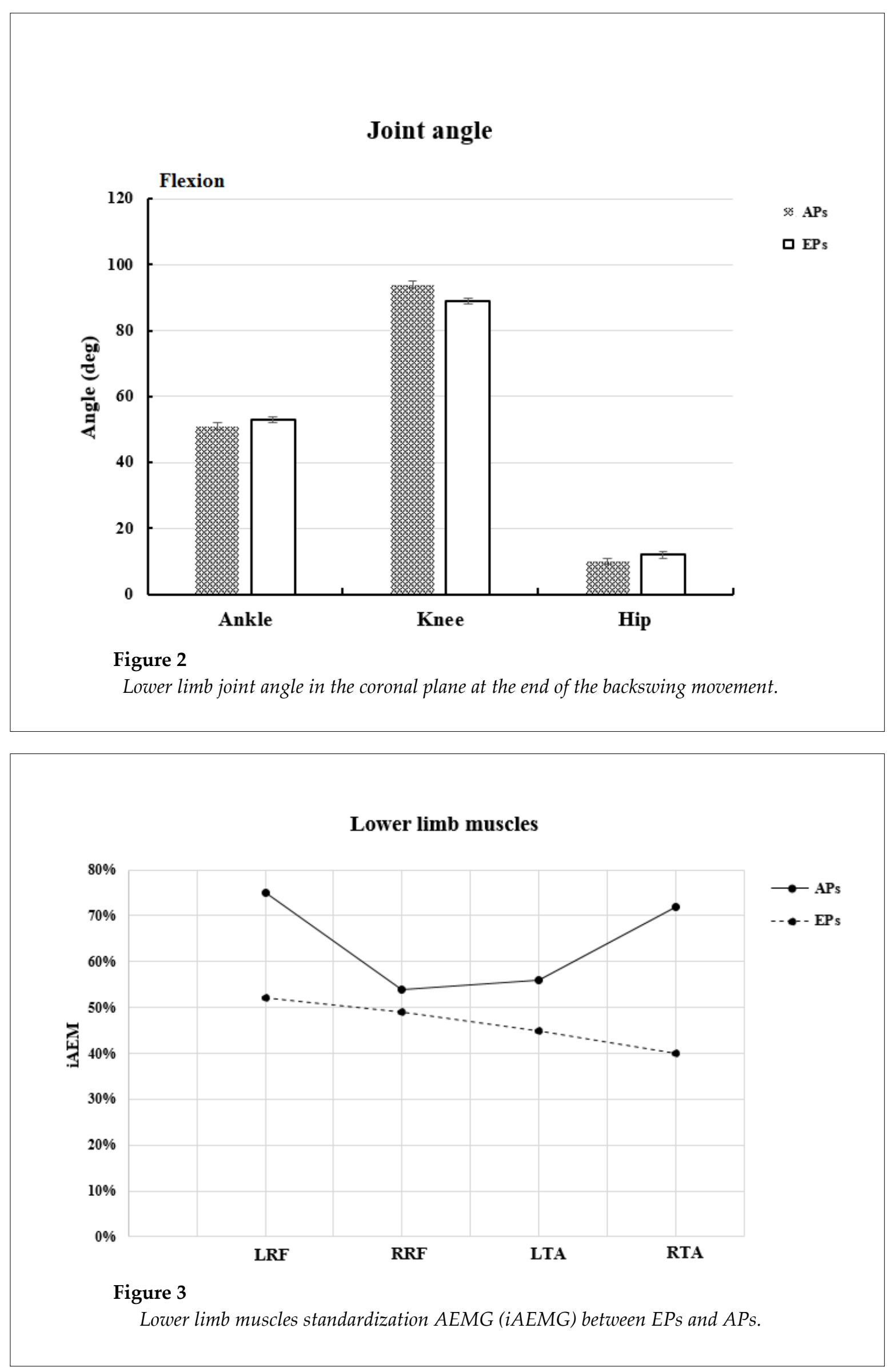

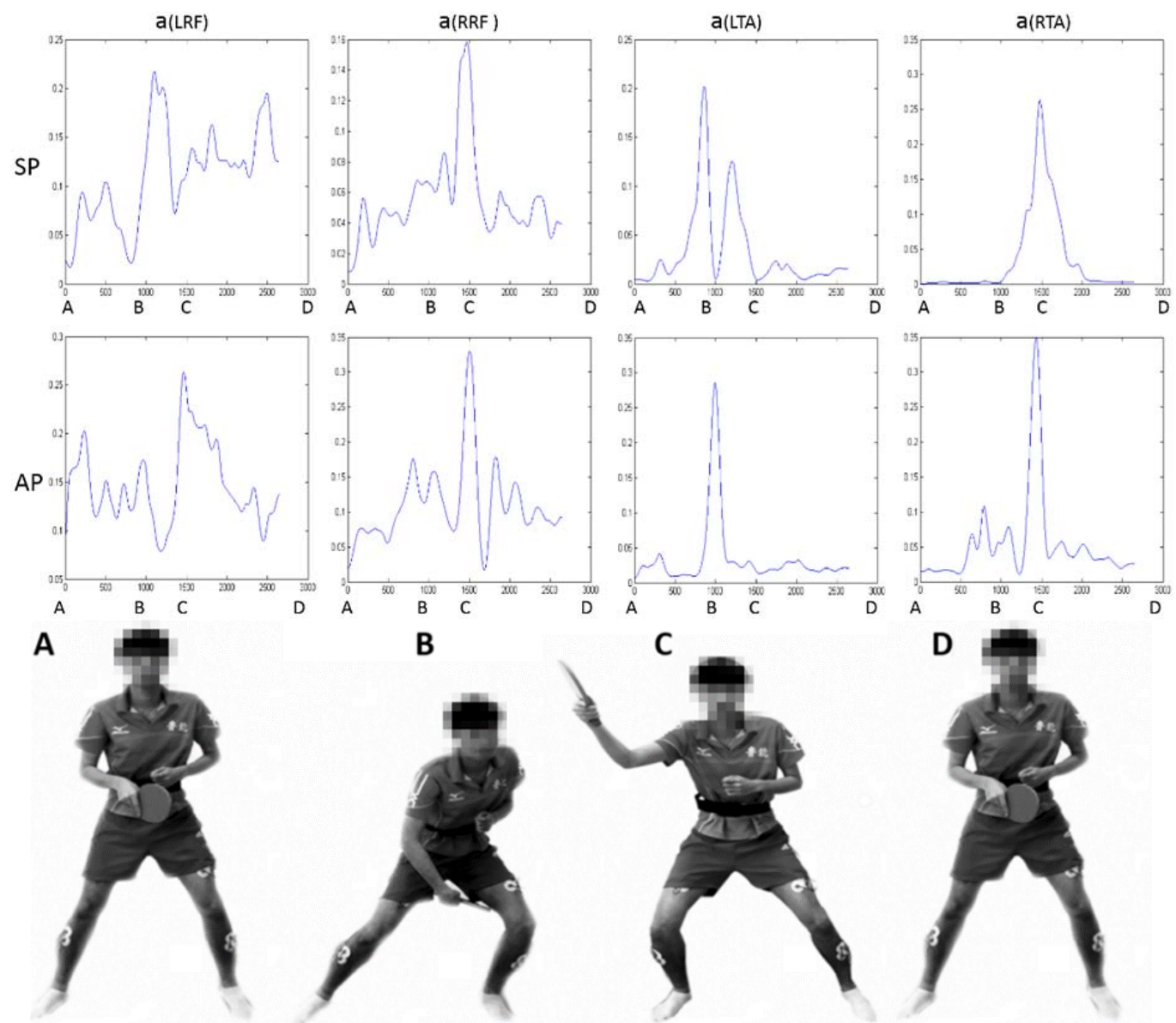

B

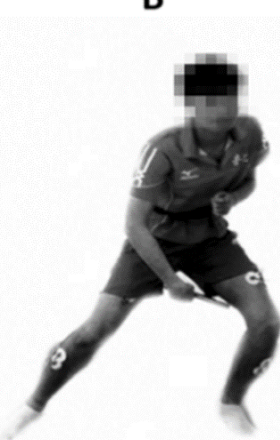

C

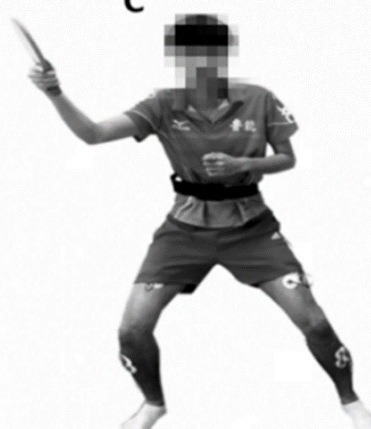

D

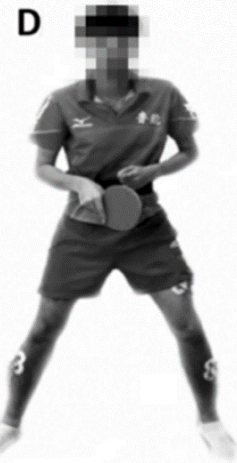

Picture 1

Muscle activation of the lower limb between EPs and APs (A represents the beginning of the backswing, $B$ represents the end of the backswing,

$C$ represents the end of the swing, $D$ represents the end position).

\section{Discussion}

The aim of this study was to investigate kinematics and electromyographic signals during table tennis topspin loop against backspin movement between elite and amateur players (EPs vs. APs). The motion was divided into two phases, backswing and swing, for data analysis. The results in this study showed that there were no significant differences in flexion of the ankle, knee and hip at the end moment of the backswing. Yet, at the beginning moment of the backswing, there were significant differences in the sagittal, coronal and horizontal planes of joints (beside the horizontal plane of the knee joint) between EPs and APs. Compared to APs, the joints of EPs showed significantly larger ankle eversion, knee flexion and hip flexion. This highlights that EPs prepare better for the stroke, which can lead to quicker shots, and shorter time of reaching the location of the swing with better adjustment of the swing action. $\mathrm{Xu}$ (2010) came to a similar conclusion. In addition, the hip joint angle variation range of EPs showed larger flexion-extension compared to APs during the swing. The use of elastic energy stored in the muscular tendon junction during the eccentric systole can enhance performance of concentric 
contractions on the basis of the elongationcontraction cycle theory (Elliott, 2006; Komi and Bosco, 1978; Walshe et al., 1998). It is possible to speculate on increased hip flexion during the swing, which can enhance the power output of the gluteus maximus during the backswing and be a potential factor in increasing the swing speed in the kinetic chain. According to the results presented in Table 3, the significantly larger ankle internal rotation of APs may cause a potential risk of the ankle sprain (Fong et al., 2012). It could be helpful for injury preventing. Greater ankle dorsiflexion and knee flexion at the end moment of the swing made the centre of gravity of EPs lower than in APs, it would decrease the time of preparation to stroke the next ball.

Flexion and extension of EPs and APs' joint angles showed a significant difference, and CMC values of EPs were all beyond 0.9, indicating high similarity of curves of their joint angle change during topspin loop against backspin movement, while the curves of APs were located within the range of moderate similarity (CMC of 0.5 to 0.75 ). This shows that EPs achieved a higher level of control and consistency of table tennis technique after long-term training and competition.

The lower extremity muscle standardization AEMG of EPs and the normalized AEMG data of EPs on both sides of the lower limb muscle were smaller compared to APs. Additionally, the maximum activation of each muscle in EPs was smaller than in APs, and MPF of the lower limbs for EPs was greater than that of APs in the whole technical movement of the backhand backspin loop, but other factors affect EMG collection, including intracellular action potential duration, should be taken into consideration.

During the swing, EPs could complete the backhand backspin loop in less time, and the speed rate of change in the joint angle was higher than that of APs. The time interval and order of momentum transfer from the proximal to the distal end were used to evaluate the quality of the whiplash-like action (Kibler and Van, 2001). Shorter pedaling time and longer pedaling distance were obtained to measure the action of lower limbs during the swing phase. Sports experts have believed that the most effective serve of high-level table tennis depends on the speed of the serve, which is largely influenced by the degree of torso twisting (Bahamonde, 2000; Elliott et al., 1995, 2003). In this study, compared to APs, EPs needed a shorter time during the swing, presented a larger joint angle range of the lower limb and a larger speed rate of joint angle change. Hence, EPs presented a faster momentum transfer and a shorter time in a lower limb pedal and stretch. In table tennis, less time to complete hitting the ball represents a higher speed of the ball. This provides benefits not only in speed, as it also provides sufficient time to move to the right position to hit the ball (Roy et al., 1998). In addition, for high level athletes, it is considered that the swing phase takes less time throughout the action cycle and is a vital factor that increases the speed in the forehand loop during the swing (Endo, 2006).

\section{Conclusion}

Compared to APs, EPs prepared better for the stroke, which led to quicker shots and shorter time to reach the location of the swing. The speed rate of joint angle change was also greater than in APs, leading to more rapid transmission of power from the lower limb to the trunk to the upper limb. Normalized AEMG data of EPs on both sides of the lower limb muscle were smaller. In addition, the maximum muscle activation observed in EPs was smaller than in APs. MPF of the lower limbs was greater for EPs in the whole technical movement of the backhand backspin loop, highlighting that EPs could complete this technical action with functional savings over APs.

\section{Acknowledgements}

The study was sponsored by the National Natural Science Foundation of China (81301600), K.C.Wong Magna Fund at the Ningbo University.

\section{References}

Bahamonde RE. Changes in angular momentum during the tennis serve. J Sport Sci, 2000; 18: 579-592 Buchanan TS, Lloyd DG, Manal K, Besier TF. Neuromusculoskeletal modeling: estimation of muscle forces 
and joint moments and movements from measurements of neural command. J Appl Biomech, 2004; 20: 367-395

Chow JW, Shim JH, Lim YT. Lower trunk muscle activity during the tennis serve. IBS J Sci, 2003; 6: 512-518

Elliott B. Biomechanics and tennis. Br J Sports Med, 2006; 40: 392-396

Elliott B, Fleisig G, Nicholls R, Escamilia R. Technique effects on upper limb loading in the tennis serve. IBS J Sci, 2003; 6: 76-87

Elliott BC, Marshall RN, Noffal GJ, Contributions of upper limb segment rotations during the power serve in tennis. J Appl Biomech, 1995; 11: 433-442

Endo M. The effectiveness of the training for the rear leg technique in tennis ground stroke. Res Phy Educ, 2006; 51: 801-815

Fong DT, Ha SC, Mok KM, Kinematics analysis of ankle inversion ligamentous sprain injuries in sports: five cases from televised tennis competitions. Am J Sports Med, 2012; 40: 2627-2632

Fu F, Zhang Y, Shao S, Ren J, Mark L, Gu Y. Comparison of center of pressure trajectory characteristics in table tennis during topspin forehand loop between superior and intermediate players. Int J Sport Sci, 2016; 11: 559-565

Gołaś A, Maszczyk A, Pietraszewski P, Stastny P, Tufano JJ, Zając A. Effects of pre-exhaustion on the patterns of muscular activity in the flat bench press. J Strength Cond Res. 2017; 31(7): 1919-1924

Iino Y, Kojima T. Effect of the racket mass and the rate of strokes on kinematics and kinetics in the table tennis topspin backhand. J Sport Sci, 2015; 34: 1-9

Kadaba MP, Ramakrishnan HK, Wootten ME. Repeatability of kinematic, kinetic, and electromyographic data in normal adult gait. J Orthop Res, 1989; 7: 849-860

Kibler WB, Van der Meer D. Mastering the kinetic chain, In: World-Class Tennis Technique. J Champaign Ill: Human Kinetics, 2001; 57: 99-113

Komi PV, Bosco C. Utilization of stored elastic energy in leg extensor muscles by men and women. Med Sci Sport, 1978; 10: 261-265

Kojima YIT. Kinematics of table tennis topspin forehands: effects of performance level and ball spin. J Sport Sci, 2009; 27: 1311-1321

Li H, Tong L, Zhou X. Comparison of Kinematic Data of Lower Extremity between Image Analysis and Real Time Motion Capture System. J Beijing sports university, 2011; 34: 126-128

Maszczyk A, Gołaś A, Pietraszewski P, Roczniok R, Zając A, Stanula A. Application of Neural and Regression Models in Sports Results Prediction. Procedia - Soci Behavio Sci, 2014; 117: 482-487

Neal RJ. The mechanics of the forehand loop and smash shots in table tennis. Aust J Sci Med, 1991; 23: 3-11

Qian J, Zhang Y, Julien B, Gu Y. Effects of performance level on lower limb kinematics during table tennis forehand loop. Acta Bioeng Biomech, 2016; 18: 149-155

Romanliu D. The influence of confounding factors on the relationship between muscle contraction level and MF and MPF values of EMG signal: a review. Int J Occup Saf Ergon, 2016; 22: 77-91

Roy SH, Bonato P, Knaflitz M. EMG assessment of back muscle function during cyclical lifting. J Electromyogr Kinesiol, 1998; 8: 233-245

Walshe AD, Wilson GJ, Ettema GJ. Stretch-shorten cycle compared with isometric preload: contributions to enhanced muscular performance. J Appl Physiol, 1998; 84: 97-106

Yoichi I, Teruaki M, Takeji K. Contributions of upper limb rotations to racket velocity in table tennis backhands against topspin and backspin. J Sport Sci, 2008; 26: 287-293

$\mathrm{Yu}$ B. Effect of external marker sets on between-day reproducibility of knee kinematics and kinetics in stair climbing and level walking. Res Sports Med, 2003; 11: 209-218

\section{Corresponding author:}

\section{Yaodong Gu}

Faculty of Sports Science, Ningbo University,

No. 818, Fenghua Road, Jiangbei District, Ningbo, Zhejiang Province, China.

E-mail: guyaodong@hotmail.com 